\title{
A possible path to a new class of ferromagnetic and half metallic ferromagnetic materials
}

\author{
I.S. Elfimov ${ }^{1}$, S. Yunoki ${ }^{1}$ and G. A. Sawatzky ${ }^{1,2}$ \\ ${ }^{1}$ Solid State Physics Laboratory, Materials Science Center, \\ University of Groningen, Nijenborgh 4, 9747 AG Groningen, The Netherlands \\ ${ }^{2}$ Department of Physics and Astronomy, University of British Columbia, \\ 6224 Agricultural Road, Vancouver, B.C. V6T 1Z1, Canada
}

(Dated: October 23, 2018)

\begin{abstract}
We introduce a path to a possibly new class of magnetic materials whose properties are determined entirely by the presence of a low concentration of specific point defects in their crystal structure. Using model Hamiltonian and ab-initio band structure methods we demonstrate that even large band gap nonmagnetic materials as simple as $\mathrm{CaO}$ can exhibit extraordinary properties like half metallic ferromagnetism upon introducing a small concentration of Ca vacancies. We show that such defects will initially bind the introduced charge carriers at neighboring sites and depending on the internal symmetry of the clusters formed by neighboring sites form "local" magnetic moments which for concentrations as low as 3\% transform this non-magnetic insulator into a half metallic ferromagnet.
\end{abstract}

The recent discovery of ferromagnetism with high transition temperatures and very small magnetic moments in the hexaborides [1], Co substituted $\mathrm{TiO}_{2}$ [2], and $\mathrm{Co}$ substituted $\mathrm{ZnO}[3]$ has opened a lively discussion regarding the role of defects in transforming insulating non magnetic compounds into ferromagnets with interesting properties In the hexaborides Monnier and Delley demonstrated, using density functional band structure approaches, that neutral $\mathrm{B}_{6}$ vacancy's in a superlattice can result in a ferromagnetic ground state even for a low density of such defects [4]. We note that the nominal valence of the $\mathrm{B}_{6}$ clusters in fairly ionic materials such as $\mathrm{CaB}_{6}$ is 2- and so two electrons are needed to charge compensate for such a B6 vacancy. It is also interesting to note that substituting divalent $\mathrm{Co}$ for a tetravalent $\mathrm{Ti}$ in $\mathrm{TiO}_{2}$ also can be compensated with two holes in the Oxygen $2 p$ valence bands which could be bound impurity states in close proximity to the Co impurity. These observations may remind some that in hydrocarbon molecules with ring structures Longuet-Higgins predicted magnetic ground states due to a kind of molecular Hund's rule coupling well known in atoms [5]. This property has in fact been used in the past by Torrance et al [6] in attempts to make organic molecular ferromagnets. More recently Eskes has used a Hubbard model calculation of ring systems and clusters to demonstrate again a kind of molecular Hund's rule coupling for orbitally degenerate states, leading to very stable magnetic (spin triplet) ground states for either two electrons or two holes, in particular geometries [7].

In the present work we report on the influence of dilute divalent cation vacancies in oxides with the rock salt structure. We predict that such systems have ferromagnetic ground states with small magnetic moments but possibly high transition temperatures. For $3.125 \% \mathrm{Ca}$ vacancies in $\mathrm{CaO}$ LDA band structure calculations predict a half metallic ferromagnetic ground state which if correct could play an important role in the modern field of spintronics and opens a path to a whole new class of ferromagnetic materials. Before presenting the band structure calculations we discuss the physics we believe to be responsible for the magnetic behaviour found. In the rock salt structure the cations are surrounded by an octahedron of anions with filled valence $p$ bands as shown in Fig.11. As also shown, the $\sigma$-bonding anion $p$ orbitals with lobes directed to the cation site, would be the most energetically favourable orbitals to accommodate the holes needed to charge compensate a cation vacancy at the center of such an octahedron. Because of the negative effective charge of the cation vacancy the holes in the anion valence $p$ band feel a strong attraction to the vacancy site and if large enough this attraction can bind two holes as in fact is found in the LDA calculations.

In order to get insight into the electronic structure of such an octaheral cluster of $\mathrm{O}^{2-}$ ions each with a full $2 p$ shell we use a tight binding like molecular orbital (MO) approach. The symmetries of the MO's formed by the $\sigma$-bonding orbitals are $a_{1 g}, t_{1 u}$, and $e_{g}$ at energies equal to $-4 t_{p p}+\epsilon_{\sigma}, \epsilon_{\sigma}, 2 t_{p p}+\epsilon_{\sigma}$ respectively. Note that simple sum of orbitals shown in Fig.1(a) represents the $a_{1 g}$ symmetry state and this state has the lowest energy for electrons and the highest for holes. The $\pi$-bonding orbitals form the symmetries $t_{2 g}, t_{1 u}, t_{2 u}$, and $t_{1 g}$ and have energies $-2 t_{p p}, 2 t_{p p \pi},-2 t_{p p \pi}$, and $2 t_{p p}$ respectively where $t_{p p}=1 / 2\left(t_{p p \sigma}-t_{p p \pi}\right)$ with the $\sigma$ and $\pi$ hopping integrals now relative to the $\mathrm{O}-\mathrm{O}$ bond direction and given in terms of electrons $\left(t_{p p \pi}\right.$ is negative and $\left.t_{p p \sigma} \sim-4 t_{p p \pi}\right)$. Note that for simplicity the zero of energy is chosen to be at $\epsilon_{\pi}$. One can see that the irreducible representation $t_{1 u}$ appears in both the $\sigma$ and $\pi$ combinations. As a result the molecular orbitals of only this particular symmetry will mix with each other resulting in a shift of their energies to $1 / 2 \epsilon_{\sigma}+t_{p p \pi} \pm 1 / 2 \sqrt{\left(\epsilon_{\sigma}-2 t_{p p \pi}\right)^{2}+32 t_{p p}^{\prime 2}}$ for the MO formed by the $\sigma("+")$ and $\pi("-")$ bonding orbitals. 

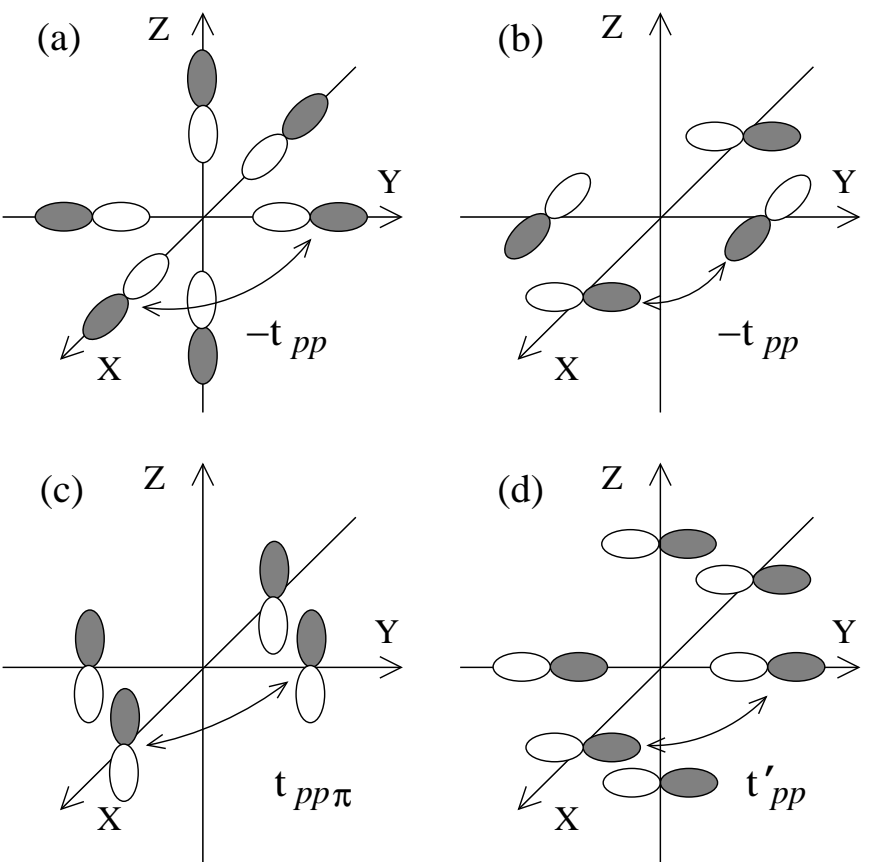

FIG. 1: An artists concept of the oxygen $\sigma$ (a) and $\pi(\mathrm{b}, \mathrm{c})$ bonding orbitals relative to the $\mathrm{O}$-vacancy bond direction surrounding a Ca vacancy. Also shown are the definitions of the hopping integrals (b) $t_{p p}$, (c) $t_{p p \pi}$, and (d) $t_{p p}^{\prime}$ given in terms of electrons.

The hopping integral $t_{p p}^{\prime}$ is defined as $1 / 2\left(t_{p p \sigma}+t_{p p \pi}\right)$ and is shown in Fig. 11(d).

The schematic energy diagram is given in Fig. 2. The lowest energy states for holes are the $e_{g}$ states formed by a linear combination of the $p$ orbitals with lobes directed to the vacancy with phases such as to result in a $e_{g}$ symmetry state when viewed from the vacancy and the $t_{1 g}$ states with a corresponding linear combination of $p$ orbitals with lobes directed perpendicular to the O-Vacancy bond direction. A simple calculation shows that a hole in $p$ orbitals with lobes pointing towards the vacancy has an energy about $1 \mathrm{eV}$ lower than a hole in a $p$-orbital with lobes perpendicular to the O-Vacancy bond direction because of the crystal field produced by the effective $2-$ charge of the cation vacancy. Therefore the lowest energy single hole state will be the doubly degenerate $e_{g}$ molecular orbital (Fig. 2(a)). We note that for anion vacancies the conduction band orbitals of the cation would be occupied by the charge compensating electrons and their energy would be lowest for the non-degenerate $a_{1 g}$ orbital as also shown in Fig.2(b).

Having understood the single-particle molecular orbitals, we move to the case of two holes (electrons) in the completely filled (empty) shell formed by the anion $p$ (cation $s$ ) orbitals in the case of cation (anion) vacancies. If the Coulomb interaction between those particles is negligible, then two holes will occupy the $e_{g}$ molecular orbital and they will form three degenerate states ${ }^{1} A_{1 g}$,

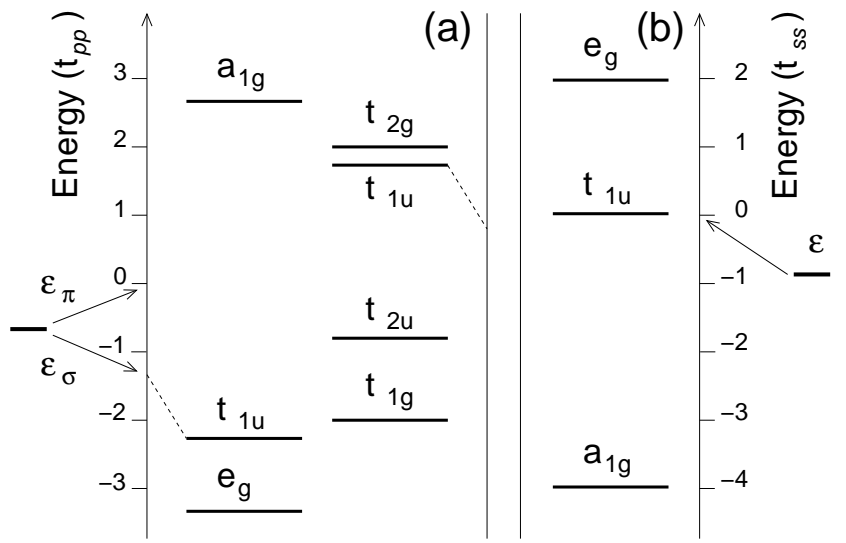

FIG. 2: Schematic single particle energy level diagram for (a) HOLES in anion orbitals and (b) ELECTRONS in cation orbitals. Dash line in fig. (a) represents a shift of energy levels of molecular orbitals with $t_{1 u}$ symmetry due to the mixture between $\sigma$ and $\pi$-bonding anion orbitals. The energy of the atomic $\sigma$ and $\pi$ orbitals are indicated by arrows on the left hand margine.

${ }^{1} E_{g}$ and ${ }^{3} A_{2 g}$ (Fig.3(b)). Two electrons on the other hand will result in a non degenerate singlet ground state (Fig.3(a)). Let us now switch on the Coulomb repulsion of which the dominant term will be that when two particles are on the same site as in a Hubbard model. The three states found for the configuration with two holes will now split up because for the singlet states the two holes can be in the same site and in the same $\sigma$ orbital while this is forbidden for the spin triplet state. This means that the singlets will increase in energy leaving us with a triplet and magnetic ground state. From Auger spectroscopy studies of a large number of simple oxides the on-site cooulomb repulsion energy of two holes in an oxygen $p$ orbital is $5-7 \mathrm{eV}$ [8, 9].

To shed more light on this issue we carried out exact diagonalization calculations for the single octahedral cluster with two electrons in $s$ orbitals as well as with two holes in the $p$ orbitals of a octahedral cluster taking into account the on-site Coulomb interaction $U$ exactly, and the results are shown in Fig. 3. One can immediately see that while a spin singlet state is the ground state for two electrons independent of the value of $U$, a spin triplet state is the ground state for the two holes as soon as $U$ is different from zero. This triplet state has symmetry ${ }^{3} A_{2 g}$ and the wave function consists mainly of a $\left(e_{g}\right)^{2}$ configuration shown schematically in Fig. 2(a). Note that the lowest singlet state is at about $0.3-0.4 \mathrm{eV}$ higher in energy than the triplet ground state of the two holes for realistic parameters. As mentioned above this is a kind of molecular Hund's rule coupling and is a result of the degenerate nature of the lowest energy molecular orbital for holes in a octahedral cluster. We note that for oxygen vacancy's it does not matter if we are dealing with $s$ or $p, d$ states of the cations. As long as band is 

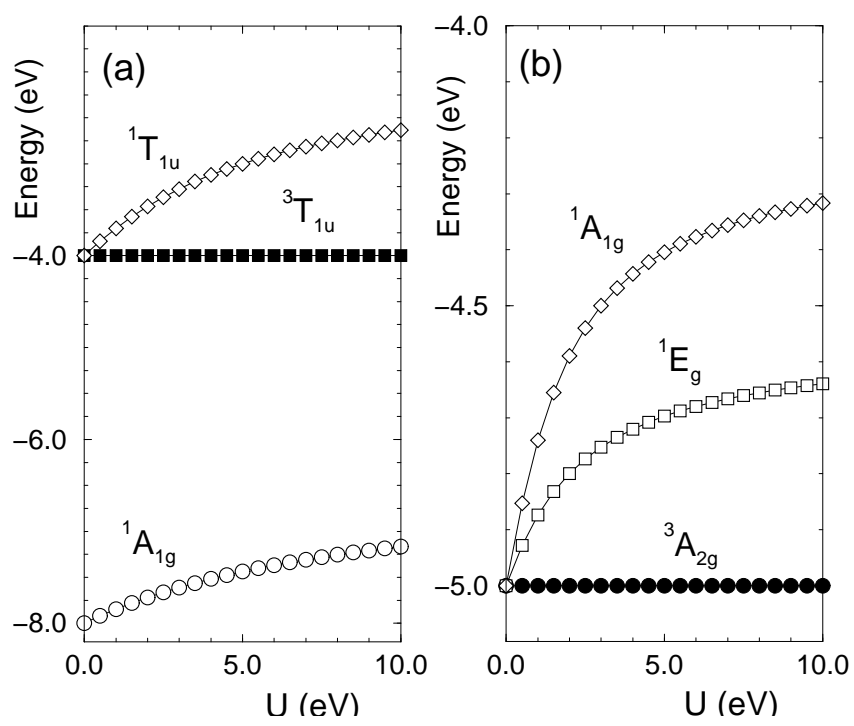

FIG. 3: Energy diagrams of the lowest three states for two particles in an octahedral cluster as a function of the on-site Coulomb repulsion $U$; (a) Two electrons introduced into the empty $s$-orbitals with the hopping integral $t_{s s}=-1.0 \mathrm{eV}$ and (b) two holes introduced into the completely filled $p$-orbitals with the hopping integrals $t_{p p \sigma}=1.2 \mathrm{eV}$ and $t_{p p \pi}=-0.3 \mathrm{eV}$ and the crystal field potential $\varepsilon_{\sigma}=1 \mathrm{eV}$ and $\varepsilon_{\pi}=0 \mathrm{eV}$ given in terms of electrons. Solid (open) symbols are for spin triplet (singlet) states.

empty the lowest energy state for electrons in octahedral geometry will always be a totally symmetric state and therefore non degenerate.

Now that we have found that cation vacancies in simple divalent monoxides with the rock salt structure or in general cation vacancies in compounds with octahedral coordination form impurity states with local magnetic moments we may ask what the magnetic state of the material is for a finite concentration of such vacancies. Do they couple ferromagnetically or antiferromagnetically and is the resulting material metallic or still insulating? To address this we consider the problem of a super lattice of cation vacancies with a $2 \times 2 \times 2$ supercell. This would correspond to a vacancy concentration of only $3.125 \%$. We calculate the electronic structure using a density functional band structure code (TBLMTO47 [10]) based on the local density approximation (LDA). For the Ca atoms, the basis from $4 s, 4 p$, and $3 d$ orbitals was used, while for oxygen, the $2 s, 2 p$ and $3 d$ states were considered. The electronic states inside the empty sphere between the atoms were expanded up to $3 d$ states including $1 s$ and $2 p$ orbitals.

Before we discuss the results of band structure calculations we note that, due to the ionic character of this compound, the valence band is formed of mainly oxygen $2 p$ orbitals and is full and the conduction band is formed of mainly $\mathrm{Ca}$ orbitals and is empty in the vacancy free compound. Pure $\mathrm{CaO}$ is therefore found to be a diamag-

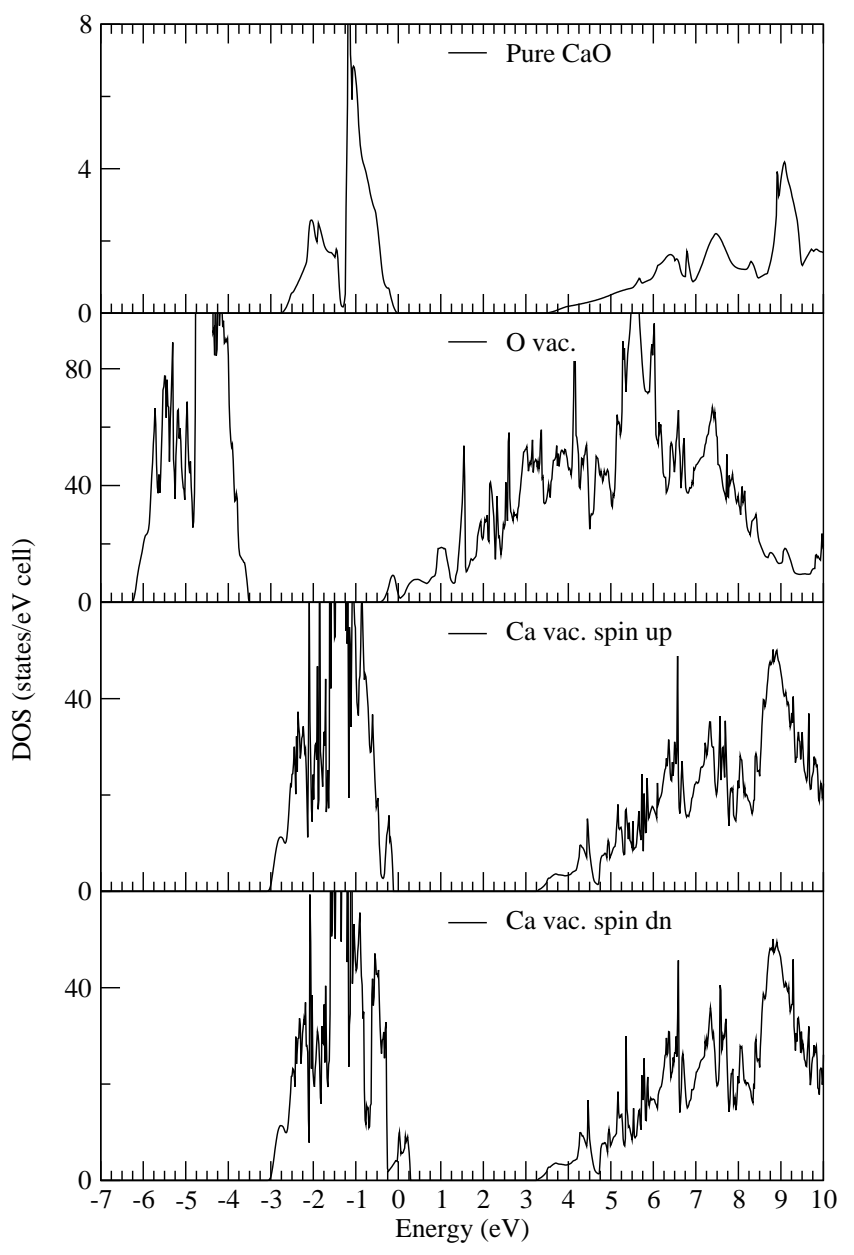

FIG. 4: Total density of states of pure CaO (upper panel) and compound with vacancy in anion (second panel) and cation (two lower panels) sublattices. The zero of energy is at Fermi energy.

netic insulator with a calculated band gap of $3.46 \mathrm{eV}$. The experimental gap is $7 \mathrm{eV}$ [11].

In Fig. 1 we display the total density of states of pure $\mathrm{CaO}$ and $\mathrm{CaO}$ with vacancies in the cation or anion sublatticies. We observe the impurity state just below the conduction band and the chemical potential shifted up above this impurity state for the case of Oxygen vacancies. This material is a non magnetic metal. The same calculation for $\mathrm{MgO}$ with Oxygen vacancies exhibits a clear gap separating the defect induced band from the conduction band resulting in an insulating non magnetic ground state. The details of calculations on $\mathrm{MgO}, \mathrm{CaO}$, and $\mathrm{SrO}$ will be presented elsewhere.

In sharp contrast the band structure for Ca vacancies exhibits also well defined defect state bands crossing the Fermi energy resulting in a metallic solution. Also here we see the clear split off character of the vacancy induced states consistent with the physical picture we presented above of the effects of the defect potential. Even more interesting is the fact that the Ca vacancy material is 


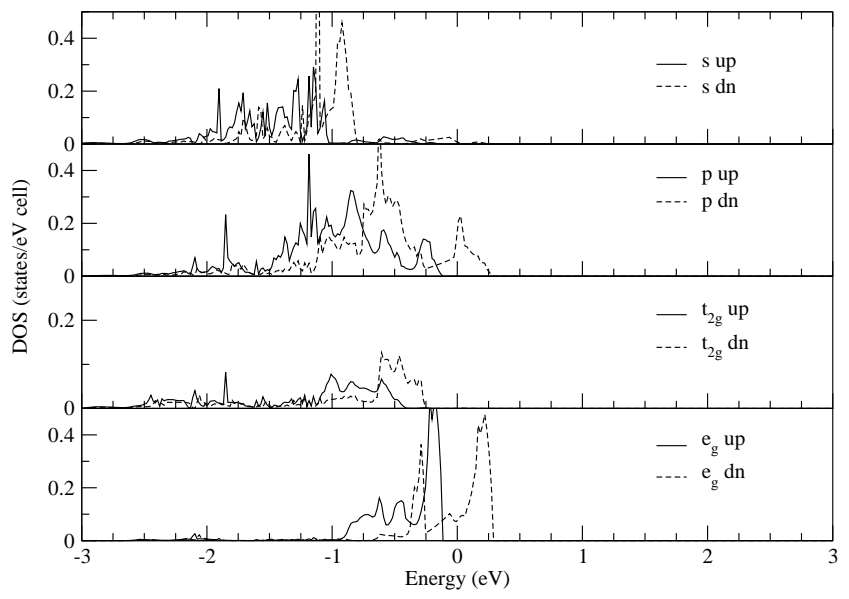

FIG. 5: Ca vacancy partial density of states. The zero of energy is at Fermi energy.

found to be ferromagnetic and the bands crossing the Fermi energy are totally spin polarized. A Ca deficiency results in a magnetic moment of $2 \mu_{B}$ per super cell, $88 \%$ of which is concentrated on the six Oxygen ions that are the nearest neighbors to the Ca vacancy.

In both cases of $\mathrm{Ca}$ or oxygen vacancies we find that the charge compensating holes or electrons are rather strongly bound to the vacancy. In Fig. 5 and Fig. 6 we show the partial density of states projected on the impurity site. It is easy to see that in the case of Ca vacancies the holes are mainly $d$ like with $e_{g}$ symmetry but band structure effects also introduce quite a strong component of $p$ symmetry at the Fermi energy resulting in a half-metallic ferromagnet. On the other hand, electrons induced by oxygen vacancies occupy local molecular orbitals of $s$ symmetry and result in a nonmagnetic ground state. Both of these agree very well with the results of our cluster calculations. We suggest that the picture presented describes a path to new classes of magnetic materials and is a very general phenomenon.

The general underlying rule for obtaining a magnetic ground state is that the crystal structure must be such that the ground state of the charge compensating molecular orbital is orbitally degenerate. Secondly the local impurity potential should be large enough to quite strongly bind the charge to the nearest neighbor atoms which is needed to validate the treatment as a cluster or molecule which then will result in the formation of a "local" moment. An on-site Coulomb interaction will then result in a high spin ground state for more than one electron or hole in such a molecular orbital which will mediate a ferromagnetic magnetic coupling between such clusters via either a superexchange or a double exchange like mechanism. We note that for the case considered above the charge compensating clusters have considerable overlap even though the concentration is only a few per cent. We also note that such degenerate ground states are common

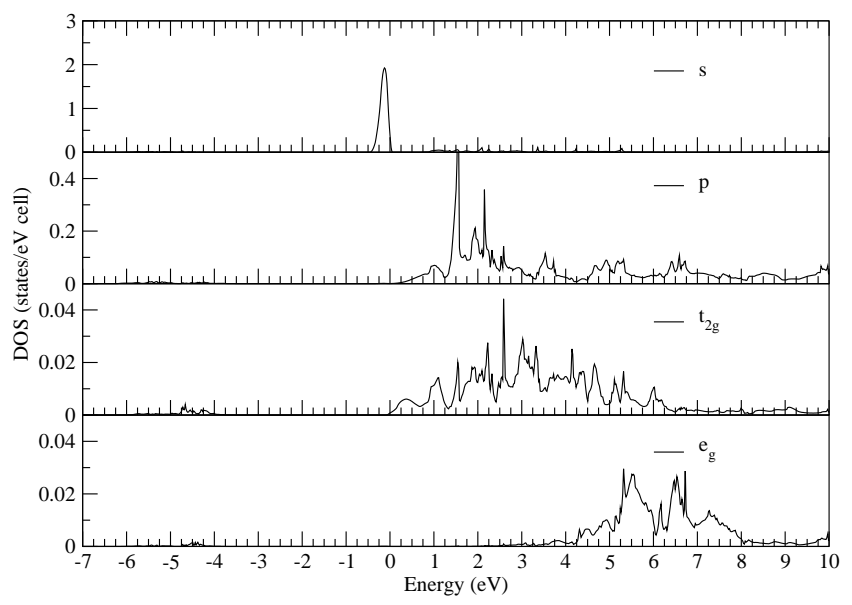

FIG. 6: Density of states projected onto the O vacancy site. The zero of energy is at Fermi energy.

for high symmetry systems such as ring like structures in addition to the octahedron considered here.

In conclusion we have demonstrated that point structural deformations of crystals such as vacancies can indeed confine the compensating charges in molecular orbitals formed by atomic orbitals on the nearest neighbours. We have shown that under certain conditions local magnetic moments will be formed due to a kind of molecular Hunds rule coupling with energetics determined by kinetic energy and symmetry considerations rather than exchange interactions. This can lead to high temperature ferromagnetic ground states and in the example discussed even a half metallic ferromagnetic ground state. Because of the rather general nature of the physics presented we believe that this may point to a path towards a new class of ferromagnetic materials.

[1] D.P. Young et al., Nature 397, 412 (1999).

[2] Y. Matsumoto et al., Science 291, 854 (2001).

[3] K.Ueda, H.Tabata and T.Kawai, Appl. Phys. Lett. 79, 988 (2001).

[4] R. Monnier and B. Delley, Phys. Rev. Lett. 87, 157204 (2001).

[5] H.C. Longuet-Higgins, J. Chem. Phys. 18, 265 (1950).

[6] J. B. Torrance et al., J. Appl. Phys. 63, 2962 (1988).

[7] H. Eskes Some Unusual Aspects of Correlated Systems, Ph.D. thesis, University of Groningen (1992).

[8] J. Ghijsen, L.H. Tjeng, J. van Elp, H. Eskes, J. Westerink, G.A. Sawatzky, and M.T. Czyzyk, Phys. Rev. B 38, 11322 (1988).

[9] S. Altieri Electronic Structure of Oxide Thin Films on Metals, Ph.D. thesis, University of Groningen (1999).

[10] O. K. Andersen, Phys. Rev. B12, 3060 (1975).

[11] R.C. Whited and W.C. Walker, Phys. Rev. 188, 1380 (1969). 